\title{
An electrically driven and readable molecular monolayer switch using a solid electrolyte
}

\author{
Elena Marchante, ${ }^{[\mathrm{a}]}$ Núria Crivillers, ${ }^{[\mathrm{a}]}$ Moritz Buhl, ${ }^{[\mathrm{a}]}$ Jaume Veciana ${ }^{[\mathrm{a}]}$ and Marta Mas-Torrent ${ }^{\star[a]}$
}

\begin{abstract}
The potential application of molecular switches as active elements in information storage has been demonstrated through numerous reported works. Importantly for devices, such switching capability has also been observed on self-assembled monolayers (SAMS). SAMs of electro-active molecules have been exploited in the last few years as electrochemical switches. Typically, the state of these switches could be read out through their optical and/or magnetic responses. These output reading processes are difficult to integrate into devices and in addition there is a need to use liquid environments to switch the redox-active molecular systems. Here, we overcome both these challenges using an ion-gel as electrolyte medium achieving an unprecedented solid state device based on a single molecular layer. Further, electrochemical impedance has been successfully exploited as the output of the system.
\end{abstract}

Bistable molecules that can be externally interconverted between two or more states with different properties have been known for a long time and their potential as active elements in information storage in future emerging devices recognized. ${ }^{[1]}$. In order to move towards device miniaturization, single molecules or a monolayer of molecules have been employed as active components in switches. In such systems, the input and output signals can be of different nature such as chemical, optical, magnetic or electrical. However, electrical signals are particularly appealing since they can be more easily integrated with current technologies. Single electroactive molecules have been incorporated in three terminal junctions, where an electrical field generated by applying a voltage to a gate terminal was employed to modulate the molecular charge, and the current through the molecule connected between two contacts was used as readout mechanism. ${ }^{[2]}$ Although scientifically extremely motivating, these devices are technologically very demanding and currently show poor fabrication yields and device reproducibility. The preparation of hybrid surfaces that consist of inorganic conducting supports functionalized with a chemically bonded self-assembled monolayer (SAM) offers a clear alternative platform for the preparation of molecular devices. ${ }^{[3]}$

The preparation of SAMs from solution, which is based on the spontaneous surface self-organization of the molecules, represents a very low cost as well as powerful and versatile tool for the modification of surfaces at the molecular level. SAMs of electroactive molecules have been exploited in the last few years as electrochemical switches. ${ }^{[4]}$ Typically, the state of these switches could be read out through their optical and/or magnetic responses. ${ }^{[5]}$ Despite the extremely encouraging results

[a] E. Marchante, Dr. N. Crivillers, M. Buhl, Prof. J. Veciana, Dr. M. Mas-Torrent

Institut de Ciència de Materials de Barcelona (ICMAB-CSIC) and CIBER-BBN, Campus de la UAB, 08193, Bellaterra, Spain E-mail: mmas@icmab.es achieved so far, two main bottlenecks have been clearly identified which seriously hamper the progress in the field towards real applications: $i)$ the reading processes that the output signals require are difficult to integrate into devices, and ii) in order to switch between the different accessible redox states of molecular systems there is an obvious need to use solutions containing an electrolyte to stabilize the charged states (i.e., ion pairing) and to ensure ionic conductivity in the electrochemical cell. Here, we overcome both these challenges employing SAMs with an electroactive ferrocene (Fc) unit and following a two-fold approach. First, electrochemical impedance spectroscopy (EIS) has been applied to measure the capacitance of the SAM and exploited as output signal of the switch. Further, a solid state device based on such a single molecular layer as active component has been successfully achieved using a solid electrolyte medium. These results represent a step forward in the field raising the perspectives of employing hybrid surfaces as molecular switches for their future integration in electronic applications.

SAMs on gold of ferrocenylalkylthiol derivatives have been established as reference systems in electrochemical studies of surface confined molecules. ${ }^{[6]}$ For this reason, SAMs of 11(ferrocenyl)undecanethiol $\left(\mathrm{FcC}_{11} \mathrm{SH}\right)$ were employed here as a switchable redox unit, since ferrocene exhibits two accessible redox states (Figure 1a). A home-made electrochemical cell was fabricated for this purpose which is shown in Figure $1 \mathrm{~b}$. Three coplanar gold electrodes (counter (CE), working (WE) and reference (RE)) were thermally evaporated through a mask on a glass substrate and a silicon mold or a Teflon ring were used to delimit a fixed area of the electrolyte in contact with the electrodes. The Au WE was modified with the ferrocene SAM by its immersion in a $1 \mathrm{mM}$ solution of the $\mathrm{FcC}_{11} \mathrm{SH}$ in ethanol for 18 hours (Figure S1).

The electrochemical properties of the SAMs were first investigated using an ionic liquid (IL) as electrolyte. Figure 1c shows the CV of FC-SAM using 1-ethyl-3-methylimidazolium bis(trifluoromethyl-sulfonyl)imide (EMIM-TFSI) as electrolyte and employing the electrochemical cell described above. A redox peak at $E^{1 / 2}=0.23 \mathrm{~V}$ ( vs Au at a scan rate of $200 \mathrm{mV} / \mathrm{s}$ ) is observed corresponding to the $\mathrm{Fc} / \mathrm{Fc}^{+}$pair. The almost insignificant voltage difference observed between the cathodic and anodic peak $(\Delta \mathrm{E}=22 \mathrm{mV}$, at $200 \mathrm{mV} / \mathrm{s})$ is characteristic of surface confined molecules, which is further confirmed with the observed linear dependence of the current density of the redox peak $\left(J_{p}\right)$ with the scan rate in the range $50-500 \mathrm{mV} / \mathrm{s}$ (Figure S2). ${ }^{[7]}$ A surface coverage of $3.7 \times 10^{-10} \mathrm{~mol} / \mathrm{cm}^{2}$ was estimated which is slightly lower than the reported theoretical value $(4.5 \mathrm{x}$ $\left.10^{-10}\right){ }^{[8]}$ The observed smaller number of electroactive sites within the SAM could be explained by the large size of the $\mathrm{TFSl}^{-}$ anion, that determines the ion pairing formation and, as reported, if it cannot fully compensate the positive charges of all $\mathrm{Fc}^{+}$, some of the Fc units would be apparently none-electroactive. ${ }^{[9]}$ 


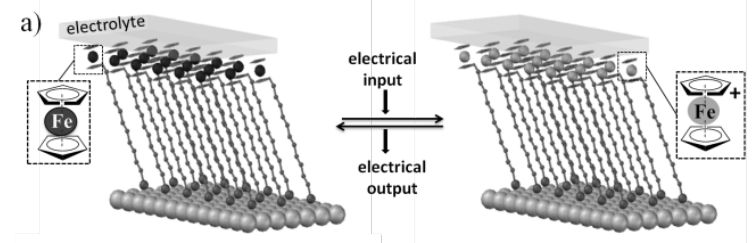

b)

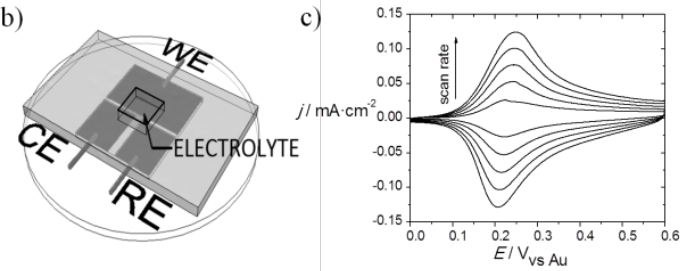

Figure 1. a) Scheme of a molecular switch based on a ferrocene SAM on gold b) Home-made electrochemical cell with three coplanar gold electrodes. c) Cyclic votammograms of the Fc-SAM at different scan rates, 100, 200, 300, 400 and $500 \mathrm{mV} / \mathrm{s}$ using the modified Au as working electrode (WE) and gold as reference and counter-electrode using the ionic liquid EMIM-TFSI.

Impedance spectroscopy (EIS) is a powerful tool to characterize electrical interfacial properties. ${ }^{[10][11][12]}$ Here, EIS is exploited to measure the capacitance of the different redox states of the switchable SAM. In an ideal situation, the gold metal surface would act as one of the capacitor plate and the physisorbed ions at the SAM/electrolyte interface would act as the other capacitor plate. ${ }^{[13]}$

EIS measurements were performed at two different applied $\mathrm{DC}$ bias, before $\left(\mathrm{V}_{0}=+10 \mathrm{mV}\right)$ and after $\left(\mathrm{V}_{1}=+450 \mathrm{mV}\right)$ the redox peak, which define the state 0 and state 1 of the switch (see supporting information for experimental details). Figure $2 a$ shows the Bode magnitude plot (impedance modulus $(|Z|)$ vs. frequency). It is clearly seen that for both states at low frequencies the plot consists of two parallel straight lines with a slope of around -0.9 . This behavior is characteristic of capacitorlike systems and the deviation from a slope of -1 can be explained by capacitance dispersion due to the adsorption of ions on the electrodes and the roughness of the electrode surface ${ }^{[14]}$ Accordingly, in the Bode phase plot ( $\varphi$ vs $f$ ) the phase angle slightly deviates from $90^{\circ}$ at low frequency, which would be expected for an ideal ionic insulator (Figure S3a). ${ }^{[12 a]}$ Remarkably, a significant decrease in $|Z|$ of one order of magnitude is observed between state 0 and state 1 at this low frequency region, indicating that the performed impedance measurements can be successfully used as a readout of the molecular switch. As a reference, the same experiments were performed in undecanethiol SAMs but no impedance differences were observed when different DC bias were applied to the functionalized WE at this frequency region (Figure S4). This indicates that the changes observed in $|Z|$ for the FC-SAMs do not come by the simple polarization of the electrode/electrolyte interface by the applied voltage but, instead, the redox state of the electroactive species is playing a crucial role.

At higher frequencies $(f>10 \mathrm{KHz})$, the frequencyindependent impedance (Figure 2a) and phase angle close to zero (Figure S3a,c) indicate that the system behaves as a resistor, that is, the total impedance is dominated by the solution resistance. ${ }^{[13]}$ This is due to the fact that at high frequencies, the capacitor behaves a short-circuit element since there is no time to be charged, allowing the AC current to pass.

The EIS measurements can also be presented in the form of real or imaginary part of the interfacial complex capacitance as a function of frequency. The real part of capacitance per unit area $\left(C_{r e}\right)$ were calculated by using the following expression: ${ }^{[15]}$

Cre $(f)=\frac{1}{2 \pi f} \frac{Z i m}{\left[\operatorname{Zre}^{2}+Z^{2}{ }^{2}\right]} \frac{1}{A}$

where $A$ is the area of the working electrode, $Z_{r e}$ and $Z_{i m}$ are the real and imaginary part of the complex impedance, respectively, and $f$ the frequency.

Figure $2 \mathrm{~b}$ shows the $C_{r e}$ versus frequency for the Fc-SAMs at both applied DC bias voltages. At high frequencies, the measured capacitance corresponds to the bulk capacitance, whereas at low frequency values a plateau is reached reflecting the interfacial capacitance.$^{[15 c]}$ As clearly observed in this figure, the $C_{r e}$ measured at $1 \mathrm{~Hz}$ is significantly different between state $O(\mathrm{~V}=10 \mathrm{mV})$ and state $1(\mathrm{~V}=450 \mathrm{mV})$. Such large on/off ratio in the capacitance measured in the two states $\left(\Delta C_{r e}=44\right.$ $\mu \mathrm{F} / \mathrm{cm}^{2}$ ) makes it possible to use this parameter to follow the switch. We attribute this difference in capacitance mainly to the charges accumulated at the electrode interface influenced by the redox state of the Fc molecules, although a certain contribution coming from structural changes in the SAM cannot be ruled out completely. ${ }^{[16]}$ Overall, it was demonstrated that the investigated surface-confined electrochemical switch can be electrically driven as well as read. a)

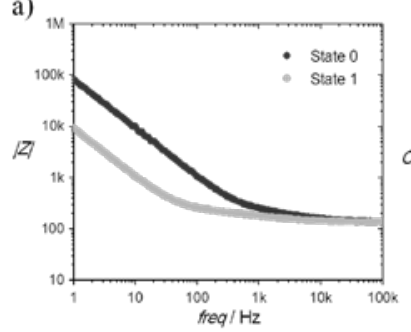

b)

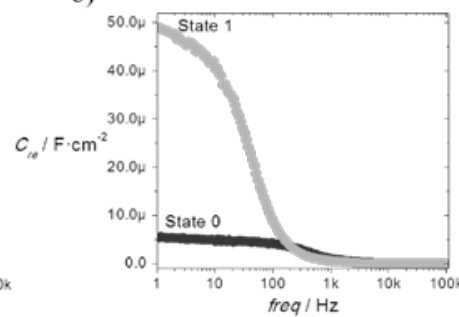

Figure 2. a) Bode magnitude plot $((|Z|)$ vs. $f)$. b) The real part of complex capacitance as a function of frequency $\left(C_{\mathrm{re}}\right.$ vs $\left.f\right)$ plot for the Fc-SAM on Au using EMIM-TFSI as electrolyte. Black line: state 0 at $+10 \mathrm{mV}$ and grey line: state 1 at $+450 \mathrm{mV}$.

A second important challenge in the field is the lack of solid state devices with such electrochemical switchable SAMs. To overcome this issue, an ionic gel (IG) has been employed as electrolyte medium. Generally, IGs are prepared by entrapping an IL in a polymer matrix. ${ }^{[17]}$ IGs have been already exploited in several electronic devices in organic electrochemical transistors $\mathrm{s},{ }^{[18]}$ as photo-actuated micro-valve $\mathrm{s}^{[19]}$ and as high-capacitance gate dielectrics. ${ }^{[20]}$ But, to the best of our knowledge, this is the first time that a solid electrolyte is employed to electrochemically control a molecular redox switch grafted on surface.

The ion-gel based on poly(vinylidene fluoride-cohexafluoropropylene) (PVDF-HFP) and EMIM-TFSI (1:4) was prepared as described in the literature (Figure S5 and S7). ${ }^{[20 a}$ 20b] The ion-gel acetone solution was drop-casted under inert atmosphere on top of the modified gold electrodes with the 
active area limited by the silicon mold. After solvent evaporation and solidification of the gel mixture the sample was removed from the glove-box and measured in air. Figure $3 a$ shows a real picture of the solid state device based on the ion-gel film.

As shown in Figure $3 \mathrm{~b}$ the $\mathrm{CV}$ electrochemical characteristics of the Fc-SAM could be successfully recorded by using the transparent, flexible and solid ion-gel on the co-planar electrodes. Remarkably the $\mathrm{CV}$ could also be measured employing a thin film of an IG that was prepared first on a glass slide, and then transferred with tweezers and laminated on top of the 3-gold coplanar electrodes with the WE modified with the ferrocene SAM. The obtained CV showed very similar features as the ones shown in Figure 3b (Figure S7).

When the CV performed with the solid electrolyte is compared with the one registered using IL, there are few electrochemical characteristics commonly used for evaluating the response of surface grafted molecules that vary. In the case of the IG, a larger peak to peak voltage difference $(\Delta E)$ as well as a more important scan rate dependence of it is observed. This is indicative of a slower electron transfer process, which is expected to occur due to the lower ionic mobility in the solid iongel film. Further, a lower current density is observed in the case of the IG despite that the density of ferrocene molecules grafted on the gold electrode should be very similar to the SAMs studied with the IL. In other words, the redox active ferrocene species electrochemically visualized is considerably lower when using the IG. This can be accounted for the less homogenous and intimate contact between the IG and the SAM as well as the less effective charge compensation of the ferrocinium molecules with the $\mathrm{TFSI}^{-}$ion, again probably due to the more limited ion diffusion in the solid state interface. This can be numerically evaluated from the integration of the $\mathrm{CV}$ redox peaks which points out that the number of molecules that participate in the electrochemical process is reduced between $30-50 \%$ from the IL to the IG.

Interestingly, EIS measurements were also performed to follow the switching process in this solid state device. In this case, state 0 and state 1 were written using a DC voltage of 10 $\mathrm{mV}$ and $600 \mathrm{mV}$ vs Au, respectively (see corresponding cyclic voltammetry in Figure S8). The impedance modulus $|Z|$ vs. frequency of the solid state device is shown in Figure 4a. Similar to before, there is a clear diminution of the impedance value when going from state 0 to state 1 , although this time the variation is smaller. Further, a noticeable difference is that in the case of the IL-based device the on/off ratio of the $|Z|$ values between the two states is maintained constant in the range 1 $100 \mathrm{~Hz}$, while in the IG device the two plots corresponding to the two states start to converge in the region $10-100 \mathrm{~Hz}$. This could again be caused by the lower mobility of the ions in the gel that prevent the formation of a compensated double layer at frequencies lower than in the IL electrolyte-SAM interface.

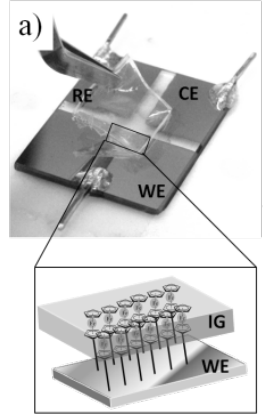

b)

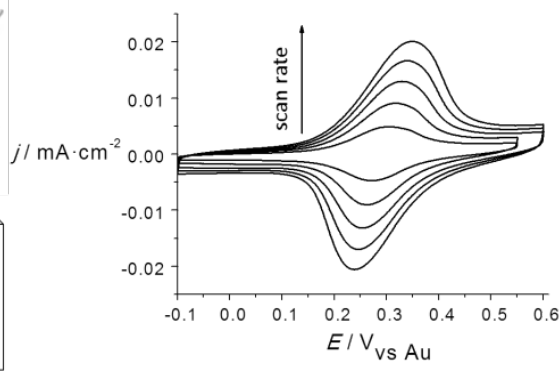

Figure 3. a) Picture of the 3-Au coplanar working (WE), counter (CE) and reference (RE) electrodes with the IG electrolyte. Scheme of the area in contact between the WE modified with the Fc-SAM and the solid electrolyte on top. b) Cyclic voltammetry response of the Au/Fc-SAM/solid ion-gel at different scan rates, 100, 200, 300, 400 and $500 \mathrm{mV} / \mathrm{s}$.

The $C_{r e}$ was calculated as explained above for the IL based switch (Figure 4b). The $C_{r e}$ values at $1 \mathrm{~Hz}$ at state 0 for the IL and IG are very similar, being $5.3 \mu \mathrm{F} / \mathrm{cm}^{2}$ and $9.25 \mu \mathrm{F} / \mathrm{cm}^{2}$ respectively. The latter value is in agreement with previous reported values for similar ion-gels. ${ }^{[20 \mathrm{~b}, 21]}$ Although the on/off ratio is lower in IG-based device than in the case of the IL one, the $\Delta C_{r e}$ is still outstanding, being $25 \mu \mathrm{F} / \mathrm{cm}^{2}$ at $1 \mathrm{~Hz}$.
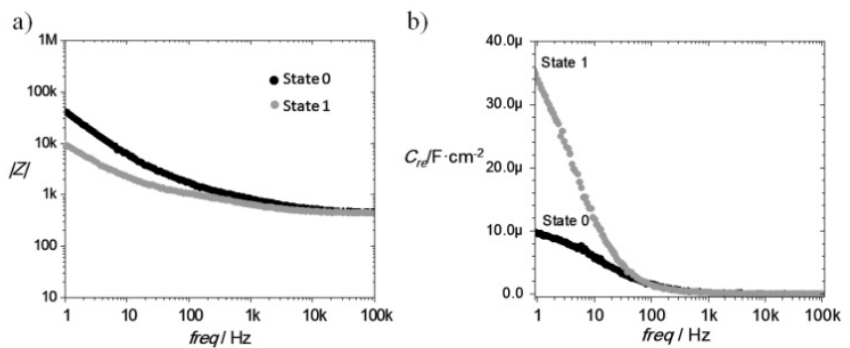

Figure 4. a) Bode magnitude plot $((|Z|)$ vs. $f)$. b) Real part of capacitance versus frequency plot for the electrochemical device, employing the solid IG as electrolyte. State $O$ (black) and state 1 (grey) correspond to DC applied bias of $10 \mathrm{mV}$ and $600 \mathrm{mV}$, respectively.

To explore the stability of the switch, 100 switching cycles were performed and, the $C_{r e}$ measured at $1 \mathrm{~Hz}$ was plotted as a function of the cycle number for both electrolyte mediums, ionic liquid (Figure 5a) and ion-gel (Figure 5b). We notice that there is a small decrease of the on/off ratio under the ionic liquid conditions which we attribute to ion permeation that can additionally cause some molecular desorption. Remarkably, although showing a lower on/off ratio, the stability of the switchable system, i.e. the switching fatigue, is improved by the use of the solid electrolyte. This demonstrates the feasibility of using the $C_{r e}$ as output of the switch even when using a solid state electrochemical device.

In summary, it has been demonstrated that with a proper design it is possible to write and read an electrochemical switch based on a self-assembled monolayer of an electroactive molecule by using an electrical input to control the switch as well as an electrical output to monitor it. This has been achieved not only using liquid environments but also in a solid state device based on an ion-gel electrolyte. The methodology employed here is simple, cheap and could be in addition extended to other 
redox-active molecules. These results represent the proof of concept of a feasible approach for the future integration of molecular monolayer-based electrochemical switches in devices.
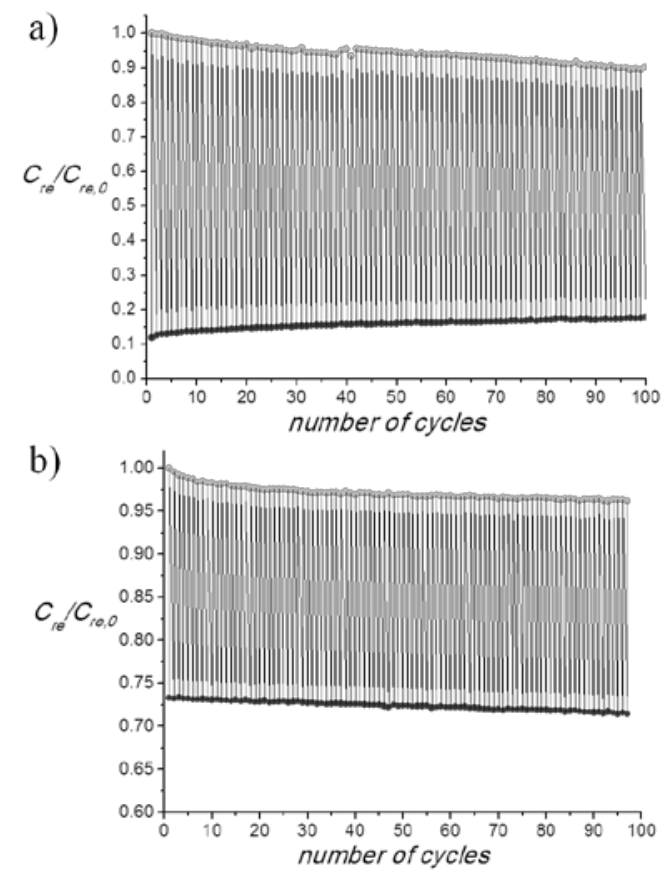

Figure 5. $C_{r e} / C_{r e, o}$ (at $1 \mathrm{~Hz}$ ) switching over 100 cycles performed alternating the applied bias with a) ionic liquid and b) ion-gel as electrolyte. In black (State $0)$ and grey (state 1).

\section{Acknowledgements}

We thank R. Pfattner for his help and F. J. del Campo for providing the silicon mold. We acknowledge the financial support of the EU projects ERC StG 2012-306826 e-GAMES, ITN iSwitch (GA no. 642196) and CIG (PCIG10-GA-2011-303989), the Networking Research Center of Bioengineering, Biomaterials and Nanomedicine (CIBER-BBN), the DGI (Spain) with project BE-WELL CTQ2013-40480-R and the Generalitat de Catalunya with project 2014-SGR-17. E. M. acknowledges the Materials Science PhD program of UAB and N.C the JdC program.

Keywords: self-assembled monolayer • ferrocene • solid electrolyte • molecular switch • electrochemical impedance spectroscopy

[1] a) J. Andréasson, U. Pischel, Chem. Soc. Rev. 2010, 39, 174-188; b) J. E. Green, J. W. Choi, A. Boukai, Y. Bunimovich, E. Johnston-Halperin, E. Delonno, Y. Luo, B. A. Sheriff, K. Xu, Y. S. Shin, H.-R. Tseng, J. F. Stoddart, J. Heath, Nature 2007, 445, 414-417; c) V. Ferri, M. Elbing, G. Pace, M. D. Dickey, M. Zharnikov, P. Samorì, M. Mayor, M. A. Rampi, Angew. Chem. Int. Ed. 2008, 47, 3407-3409.

[2] a) C. Huang, A. V. Rudnev, W. Hong, T. Wandlowski, Chem. Soc. Rev. 2015, 44, 889-901; b) M. L. Perrin, E. Burzurí, H. S. J. van der Zant, Chem. Soc. Rev. 2015, 44, 902-919; c) N. Darwish, A. C. Aragonès, T. Darwish, S. Ciampi, I. Díez Pérez, Nano Lett. 2014, 14, 7064-7070.
[3] a) V. Balzani, A. Credi, M. Venturi, ChemPhysChem 2008, 9, 202-220; b)M. Mas-Torrent, C. Rovira, J. Veciana, Adv. Mater. 2013, 25, 462-468; c) B. K. Pathem, S. Claridge, Y. B. Zheng, P. S. Weiss, Annu. Rev. Phys. Chem.2013, 64, 605-630

[4] a) Z. Liu, A. A. Yasseri, J. S. Lindsey, D. F. Bocian, Science 2003, 302, 1543-1545; b) G. Periyasamy, R. D. Levine, F. Remacle, Aust. J. Chem. 2010, 63, 173-183.

[5] a) G. de Ruiter, M. E. van der Boom, Angew. Chem. Int. Ed. 2012, 51, 8598-8601; b) A. D. Shukla, A. Das, M. E. van der Boom, Angew. Chem. Int. Ed. 2005, 44, 3237-3240; c)C. Simão, M. Mas-Torrent, J. Casado-Montenegro, F. Otón, J. Veciana, C. Rovira, J. Am. Chem. Soc. 2011, 133, 13256-13259; d)C. Simão, M. Mas-Torrent, N. Crivillers, V. Lloveras, J. M. Artés, P. Gorostiza, J. Veciana, C. Rovira, Nat. Chem. 2011, 3, 359-364.

[6] A. V. Rudnev, U. Zhumaev, T. Utsunomiya, C. Fan, Y. Yokota, K.-i. Fukui, T. Wandlowski, Electrochim. Acta 2013, 107, 33-44.

[7] H. Ju, D. Leech, Phys. Chem. Chem. Phys. 1999, 1, 1549-1554.

[8] C. A. Nijhuis, W. F. Reus, G. M. Whitesides, J. Am. Chem. Soc. 2009, 131, 17814-17827

[9] Q.-W. Sun, K. Murase, T. Ichii, H. Sugimura, J. Electroanal. Chem. 2010, 643, 58-66.

[10] E. Impedance Spectroscopy: Theory, and Applications, 2nd Edition; ISBN: 978-0-471-64749-2; Wiley 2005; Evgenij Barsoukov (Editor), J. Ross Macdonald (Editor).

[11] a)R. E. Ruther, Q. Cui, R. J. Hamers, J. Am. Chem. Soc. 2013, 135, 5751-5761; b)P. Brooksby, K. Anderson, A. Downard, A. Abell, J. Phys. Chem. C 2011, 115, 7516-7526; c)B. P. G. Silva, D. Z. de Florio, S. Brochsztain, J. Phys. Chem. C 2014, 118, 4103-4112.

[12] a) E. Boubour, R. B. Lennox, J. Phys. Chem. B 2000, 104, 9004-9010; b) W. Wang, S. Zhang, P. Chinwangso, R. C. Advincula, T. R. Lee, J. Phys. Chem. C 2009, 113, 3717-3725; c) D. K. Peng, S. T. Yu, D. J. Alberts, J. Lahann, Langmuir 2007, 23, 297-304; d) C. A. Nijhuis, B. A. Boukamp, B. J. Ravoo, J. Huskens, D. N. Reinhoudt, J. Phys. Chem. C 2007, 111, 9799-9810. [13] E. Boubour, R. B. Lennox, Langmuir 2000, 16, 4222-4228.

[14] a) T. Pajkossy, Electroanal. Chem 1994, 364, 111-125; b) Z. Kerner, T. Pajkossy, Electrochim. Acta 2000, 46, 207-211; c) T. Pajkossy, Solid State Ionics 2005, 176, 1997-2003.

[15] a) E. Katz, O. Lioubashevsky, I. Willner, J. Am. Chem. Soc. 2004, 126, 15520-15532; b) T. M. Nahir, E. F. Bowden, Langmuir 2002, 18, 5283-5296; c) C. R. Mariappan, T. P. Heins, B. Roling, Solid State Ionics 2010, 181, 859-863. [16] N. Darwish, P. K. Eggers, S. Ciampi, Y. Zhang, Y. Tong, S. Ye, M. N. Paddon Row, J. J. Gooding, Electrochem. Commun. 2011, 13, 387-390.

[17] a) M. J. Park, I. Choi, J. Hong, O. Kim, J. Appl. Polym. Sci. 2013, 129 , 2363-2376.

[18] D. Khodagholy, V. F. Curto, K. J. Fraser, M. Gurfinkel, R. Byrne, D. Diamond, G. G. Malliaras, F. Benito-Lopez, R. M. Owens, J. Mater. Chem. 2012, 22, 4440-4443

[19] F. Benito-Lopez, R. Byrne, R. A. M., N. E. Vrana, G. McGuinness, D. Diamond, Lab Chip 2010, 10, 195-201.

[20] a) K. H. Lee, M. S. Kang, S. Zhang, Y. Gu, T. P. Lodge, C. D. Frisbie, Adv. Mater. 2012, 24, 4457-4462; b) K. H. Lee, S. Zhang, T. P. Lodge, C. D. Frisbie, J. Phys. Chem. B 2011, 115, 3315-3321; c) B. J. Kim, H. Jang, S.-K. Lee, B. H. Hong, J.-H. Ahn, J. H. Cho, Nano Lett. 2010, 10, 3464-3466.

[21] S. Zhang, K. H. Lee, C. D. Frisbie, T. P. Lodge, Macromolecules 2011, 44, 940-949. 